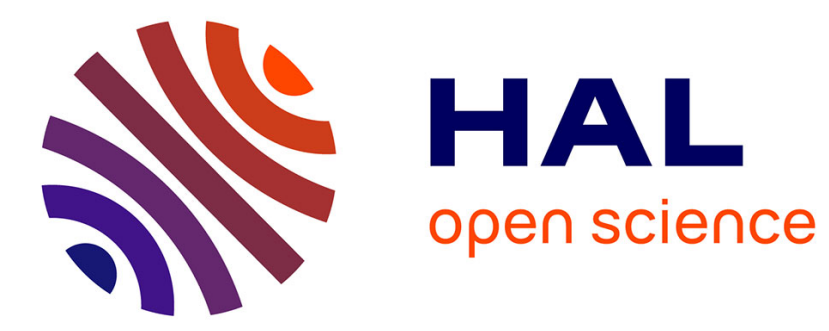

\title{
Regiospecific synthesis of neuroprotective 1,4-benzoxazine derivatives through a tandem oxidation-Diels-Alder reaction
}

Khac Minh Huy Nguyen, Leslie Schwendimann, Pierre Gressens, Martine Largeron

\section{To cite this version:}

Khac Minh Huy Nguyen, Leslie Schwendimann, Pierre Gressens, Martine Largeron. Regiospecific synthesis of neuroprotective 1,4-benzoxazine derivatives through a tandem oxidation-Diels-Alder reaction. Organic \& Biomolecular Chemistry, 2015, 13 (12), pp.3749-3756. 10.1039/c5ob00049a . hal02384706

\section{HAL Id: hal-02384706 \\ https://hal.science/hal-02384706}

Submitted on 21 Nov 2020

HAL is a multi-disciplinary open access archive for the deposit and dissemination of scientific research documents, whether they are published or not. The documents may come from teaching and research institutions in France or abroad, or from public or private research centers.
L'archive ouverte pluridisciplinaire HAL, est destinée au dépôt et à la diffusion de documents scientifiques de niveau recherche, publiés ou non, émanant des établissements d'enseignement et de recherche français ou étrangers, des laboratoires publics ou privés. 


\section{Organic \& Biomolecular Chemistry}

\section{Accepted Manuscript}

This article can be cited before page numbers have been issued, to do this please use: K. M. H. Nguyen,

L. Schwendimann, P. Gressens and M. Largeron, Org. Biomol. Chem., 2015, DOI: 10.1039/C5OB00049A.

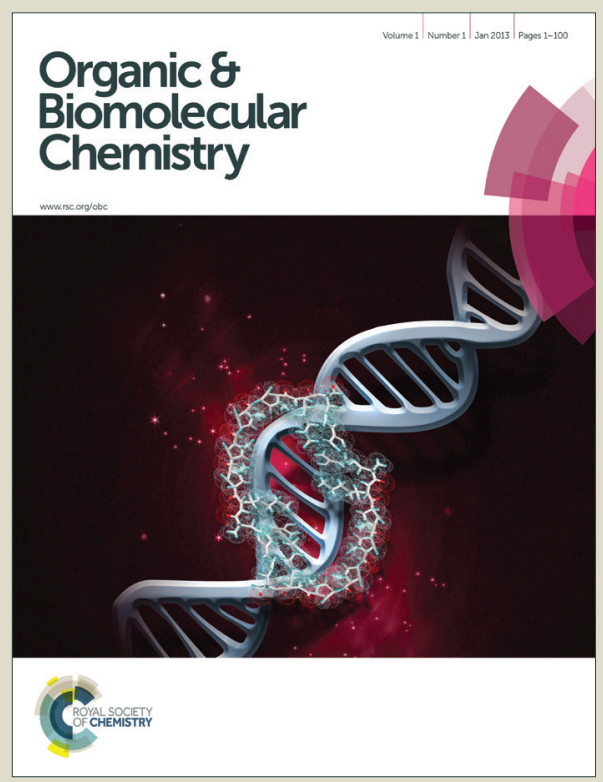

This is an Accepted Manuscript, which has been through the Royal Society of Chemistry peer review process and has been accepted for publication.

Accepted Manuscripts are published online shortly after acceptance, before technical editing, formatting and proof reading. Using this free service, authors can make their results available to the community, in citable form, before we publish the edited article. We will replace this Accepted Manuscript with the edited and formatted Advance Article as soon as it is available.

You can find more information about Accepted Manuscripts in the Information for Authors.

Please note that technical editing may introduce minor changes to the text and/or graphics, which may alter content. The journal's standard Terms \& Conditions and the Ethical guidelines still apply. In no event shall the Royal Society of Chemistry be held responsible for any errors or omissions in this Accepted Manuscript or any consequences arising from the use of any information it contains. 


\title{
Regiospecific synthesis of neuroprotective 1,4-benzoxazine derivatives through a tandem oxidation-Diels-Alder reaction
}

\author{
Khac Minh Huy Nguyen, ${ }^{a}$ Leslie Schwendimann, ${ }^{b, c}$ Pierre Gressens ${ }^{b, c}$ and Martine Largeron $*^{a}$ \\ Received (in $X X X, X X X) X$ th $X X X X X X X X X 20 X X$, Accepted Xth $X X X X X X X X X 20 X X$ \\ ${ }_{5}$ DOI: 10.1039/b000000x
}

The tandem oxidation-inverse electron demand Diels-Alder reaction of $o$-aminophenol derivatives and enamines has been accomplished at room temperature using a stoichiometric amount of manganese dioxide as the oxidant to furnish highly substituted 1,4-benzoxazine cycloadducts with complete regiochemical control. Because of its efficiency for introducing diversity elements in both cycloadditions

10 partners, this one-pot process should allow the assembly of libraries of biologically relevant 1,4benzoxazine derivatives. In this respect, 3,3-diphenyl-substituted-1,4-benzoxazine derivative $\mathbf{3 n}$ was found to be a potent neuroprotective agent in an animal model of excitotoxic lesions in newborn mice.

\section{Introduction}

1,4-Benzoxazine derivatives are an important class of 15 heterocyclic compounds because of their natural occurrence and important biological activities. ${ }^{1}$ They are potential drugs for treating infections, diabetes, asthma, depression as well as neurodegenerative, autoimmune, inflammatory and cardiovascular disorders (Figure 1). ${ }^{2}$ Traditional syntheses of 1,420 benzoxazine derivatives involve multistep processes, using 2nitrophenols or 2-aminophenols as the starting materials., ${ }^{1,3}$ However, the increasing interest in 1,4-benzoxazine derivatives has prompted to develop new concise methodologies that follow diverse one-pot strategies including metal-catalyzed reactions, ${ }^{4}$

25 cascade (or tandem) reactions ${ }^{5}$ and microwave-assisted reactions. ${ }^{6}$

A few years ago, we reported an electrochemically induced tandem oxidation-Diels-Alder reaction for the synthesis of highly functionalized 1,4-benzoxazine and 1,4-benzodioxin derivatives. ${ }^{7}$ 30 In particular, the anodic oxidation of $o$-aminophenol derivatives produced unstable $o$-quinone-monoimine heterodienes, which were trapped in situ by enamine dienophiles through regiospecific inverse-electron-demand Diels-Alder (IEDDA) reactions to give diverse 1,4-benzoxazine derivatives. Interestingly, some of these 35 compounds proved to be effective neuroprotective agents both in vitro and in vivo. ${ }^{8}$ Nevertheless, it seemed doubtful that this electrochemically induced process will ever be able to compete with more conventional chemical approaches at a preparative scale. However, the instability of $o$-quinone-monoimines 40 prohibited their efficient use as heterodienes in chemically induced IEDDA reactions. For a long time, such reactions have been mainly restricted to stable $o$-quinone-monooximes ${ }^{9}$ and $o$ quinone-monoimides. ${ }^{5 \mathrm{a}-\mathrm{d}, 5 \mathrm{f}, 10}$ Recently, the synthesis of $1,4-$ benzoxazine derivatives has been reported for the first time from 45 in situ chemically generated unstable o-quinone-monoimine heterodienes, using diacetoxy iodobenzene as the oxidizing reagent, in the presence of vinyl ether dienophiles. ${ }^{11}$ Inspired by this protocol, we looked for a chemical extrapolation of our previous electrochemical procedure.

50 Activated manganese dioxide is known as a mild oxidant in organic chemistry. Especially, it has been successfully used in combination with a nucleophilic trapping agent in tandem oxidation processes (TOP) for the synthesis of diverse heterocycles. ${ }^{12}$ As an extension of this chemistry, we report 55 herein that a stoichiometric amount of activated $\mathrm{MnO}_{2}$, in deaerated methanol, is sufficient to convert various $o$ aminophenol derivatives, in the presence of enamine dienophiles, to highly functionalized 1,4-benzoxazine derivatives through a tandem oxidation-IEDDA reaction under mild conditions. The 60 synthetic potential of this reaction has been further explored with the ultimate goal of developing libraries of biologically relevant 1,4-benzoxazine derivatives.

65

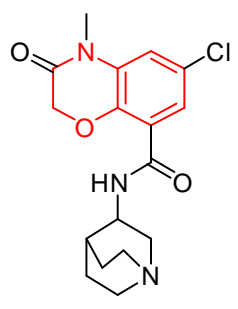

Azasetron (Serotonin 3 receptor antagonist)<smiles>C[C@H]1COc2c(N3CCN(C)CC3)c(F)cc3c(=O)c(C(=O)O)cn1c23</smiles>

Levofloxacin (Antibiotic)
Fig. 1 Example of important pharmaceutically active compounds possessing the 1,4-benzoxazine core. 


\section{Results and Discussion}

\section{Choice of the optimal reaction conditions}

Our studies began by examining the oxidation of $o$-aminophenol $\mathbf{1 a}$ in deaerated methanol, in the presence of enamine 2a. One 5 equivalent of $\mathbf{1 a}$ and 1.5 equiv of enamine $\mathbf{2 a}$, together with a stoichiometric amount of $\mathrm{MnO}_{2}$ as the oxidant, were a good reagent combination for the reaction. However, the reaction progressed slowly (entry 1, Table 1), leading after 6 h to $46 \%$ of 1,4-benzoxazine $\mathbf{3 a}$ as a single regioisomer. As previously 10 observed from electrochemical studies, ${ }^{7}$ the basicity of the enamine dienophile 2a was not sufficient to produce significant amounts of the phenolate anion of 1a, that is the sole form that can be oxidized to the $o$-quinone-monoimine species. Accordingly, when morpholine was added to the bulk solution as 15 the ancillary base (entries 2 and 3), the reaction could go to completion by acid-base equilibrium displacement. Using 1.5 equiv of morpholine, the yield of $\mathbf{3 a}$ reached $75 \%$ in only $3 \mathrm{~h}$ (entry 3). Various temperatures and solvents were further screened (entries 4-7), but none of the changes led to an 20 improvement of the yield of the cycloadduct 3a. As already observed, ${ }^{7}$ strong solvation of methanol to the $o$-quinonemonoimine species may be required to enhance the electrophilicity of its quinonoid moiety, making it able to participate in IEDDA reaction.

25 Table 1 Optimization of the reaction conditions ${ }^{a}$<smiles>CC(=O)c1ccc(O)c(N)c1O</smiles>

1a

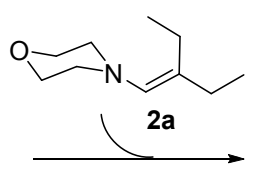

$\mathrm{MnO}_{2}$ (1 equiv) solvent, $t^{\circ} \mathrm{C}$, time $(\mathrm{h})$<smiles>CCC1(CC)Nc2c(ccc(C(C)=O)c2O)OC1N1CCOCC1</smiles>

\begin{tabular}{llllll}
\hline Entry & $\begin{array}{l}\text { Morpholine } \\
\text { (equiv) }\end{array}$ & Solvent & $\begin{array}{l}\text { Temp } \\
\left({ }^{\circ} \mathrm{C}\right)\end{array}$ & $\begin{array}{l}\text { Time } \\
(\mathrm{h})\end{array}$ & $\begin{array}{l}\text { Yield } \\
(\%)^{b}\end{array}$ \\
\hline 1 & 0 & $\mathrm{MeOH}$ & 25 & 6 & 46 \\
2 & 1 & $\mathrm{MeOH}$ & 25 & 6 & 70 \\
3 & 1.5 & $\mathrm{MeOH}$ & 25 & 3 & 75 \\
4 & 1.5 & $\mathrm{MeOH}$ & 10 & 3 & 51 \\
5 & 1.5 & $\mathrm{MeOH}$ & 40 & 3 & 44 \\
6 & 1.5 & $\mathrm{CH}_{3} \mathrm{CN}$ & 25 & 3 & 34 \\
7 & 1.5 & $\mathrm{THF}$ & 25 & 3 & 36
\end{tabular}

${ }^{a}$ Reactions were performed with 1a $(0.5 \mathrm{mmol}), 2 \mathrm{2a}(0.75 \mathrm{mmol}, 1.5$ equiv) and $\mathrm{MnO}_{2}(0.5 \mathrm{mmol}, 1$ equiv), in $25 \mathrm{~mL}$ of deaerated solvent. ${ }^{30}{ }^{b}$ Yields refer to chromatographically pure isolated products.

\section{Scope of the tandem oxidation-IEDDA process.}

The optimized reaction conditions (Table 1, entry 3) were first applied to a series of aminophenol derivatives 1a-i, in the 35 presence of alkylenamine 2a. Table 2 shows some examples of the molecular diversity that can be obtained with this reaction sequence that includes a regiospecific IEDDA reaction between the in situ generated electron-poor o-quinone-monoimine heterodiene and the electron-rich tertiary alkylenamine dienophile ${ }_{40} \mathbf{2 a}$.

Table 2 Variation of the diene part: tandem oxidation-IEDDA reaction of diverse aminophenol derivatives $\mathbf{1 a - i}$ with enamine $\mathbf{2} \mathbf{a}^{a}$
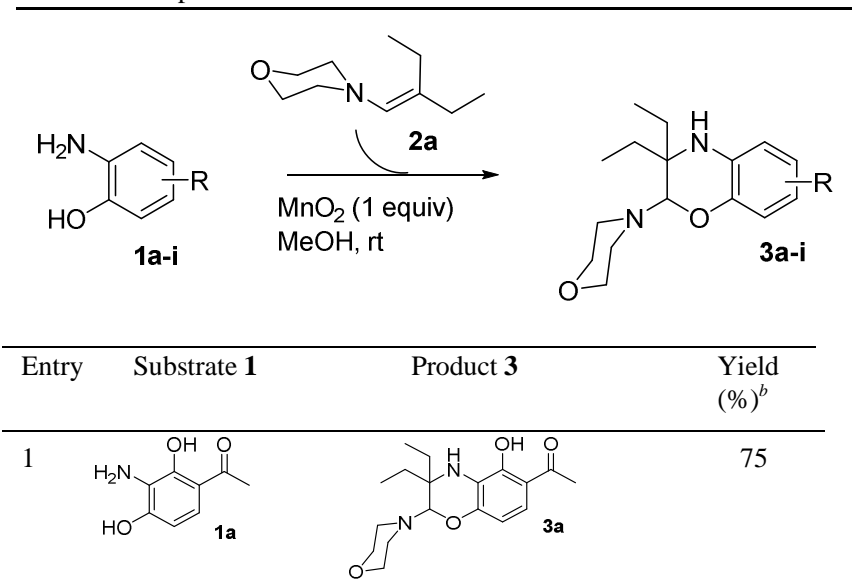

2

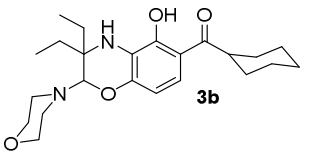

3<smiles>CC(C)CC(=O)c1ccc(O)c(N)c1O</smiles><smiles>CCC1(CC)Nc2c(ccc(C(=O)CC(C)C)c2O)OC1/N=C/C=O</smiles>

4<smiles>CC(=O)c1ccc(O)c(N)c1</smiles><smiles>CCC1(CC)Nc2cc(C(C)=O)ccc2OC1/C=C/O</smiles>

5<smiles>N#Cc1ccc(O)c(N)c1</smiles><smiles>CCC1(CC)Nc2cc(C#N)ccc2OC1N(CCO)CCO</smiles>

6<smiles>COC(=O)c1ccc(O)c(N)c1</smiles><smiles>CCC1(CC)Nc2cc(C(=O)OC)ccc2OC1/N=C/C=O</smiles><smiles>Nc1cc(C(=O)c2ccccc2)ccc1O</smiles><smiles>CCC1(CC)Nc2cc(C(=O)c3ccccc3)ccc2OC1/C=C/O</smiles>

8<smiles>CC(C)CNC(=O)c1ccc(O)c(N)c1</smiles><smiles>CCC1Nc2cc(C(=O)NCC(C)C)ccc2OC1N(C=C=O)CC</smiles><smiles>Nc1ccccc1O</smiles>
1<smiles>CCC1(CC)Nc2ccccc2OC1/C=C/O</smiles>

${ }^{a}$ Reactions were performed with 1a-i $(0.5 \mathrm{mmol}), 2 \mathrm{a}(0.75 \mathrm{mmol}, 1.5$ 45 equiv) and $\mathrm{MnO}_{2}(0.5 \mathrm{mmol}, 1$ equiv) in $25 \mathrm{~mL}$ of deaerated $\mathrm{MeOH}$, at room temperature, for $3 \mathrm{~h}$. 1.5 Equiv of morpholine was added to the bulk solution for generating the phenolate ion of 1a-i. ${ }^{b}$ Yields refer to chromatographically pure isolated products. 
The more electron-rich carbon atom of enamine dienophile $\mathbf{2 a}$ added to the nitrogen atom of the $o$-quinone-monoimine heterodiene. In all cases (Table 2, entries 1-9), the method was successful leading to the 1,4-benzoxazine derivatives in 5 acceptable yields ranging from 58 to $75 \%$. Unexpectedly, $o$ aminophenol 1i (entry 9) proved to be as reactive as electron-poor aminophenol derivatives 1a-f. This result could indicate that the electron-rich enamine $\mathbf{2 a}$ was the more active participant in the IEDDA reaction. However, when electron-rich $o$-aminophenol 10 bearing methyl or methoxy groups were oxidized in the presence of enamine $\mathbf{2 a}$ no identifiable products were obtained under the same experimental conditions.

In a second series of experiments, diverse enamine dienophiles 2a-i were screened in the presence of aminophenol 1a (Table 3). 15 As earlier shown, the use of 3,3-disubstituted enamines 2 was required for the preparation of 1,4-benzoxazine derivatives in acceptable yields. ${ }^{13}$ As expected, tertiary alkylenamines $\mathbf{2 a - c}$ with a pronounced electron-rich character produced the desired cycloadduct in high yield (entries 1-3), whereas introduction of 20 phenyl substituents resulted in lower yields (entries 4 and 5). The cycloaddition reaction could also be extended to less stable secondary enamines $\mathbf{2 f - h}$ and afforded the 1,4-benzoxazine derivatives 3n-p (entries 6-8) without further elimination of the 2-alkylamino chain, in contrast to what had been previously 25 reported for similar cycloaddition reactions of enamines with heterodienes. ${ }^{14}$ Despite of its aromaticity, commercially available 2,3-dihydro- $1 H$-cyclopent[b]indole $\mathbf{2 i}$ with latent enamine functionality could also participate in the IEDDA reaction giving the indolobenzoxazine ring system in $30 \%$ yield (entry 9).

\section{${ }_{30}$ In vivo biological evaluation of compound $3 \mathbf{n}$.}

In a previous work, we had shown that introduction of 3-phenyl substituents was crucial for efficient neuroprotective activity without the manifestation of intrinsic toxicity using in vitro assays on murine HT-22 hippocampal cell cultures. From these 35 results, we had identified a potential candidate A (Figure 2) that proved to be active in vivo in newborn mice. ${ }^{8}$ Brain lesions induced in newborn mice by the glutamatergic agonist $S$ bromowillardiine acting on kainate receptors mimic some aspects of white matter cysts and transcortical necrosis observed in 40 human perinatal brain damage, especially in the case of cerebral palsy. $^{15}$

Our present investigation into the substrate scope led us to select another candidate $\mathbf{3 n}$. The neuroprotective effects of benzoxazine $\mathbf{3 n}$ were assessed and compared with those of 45 compound $\mathrm{A}$, together with reference benzoxazines $\mathrm{B}$ and $\mathrm{C}$, two established neuroprotective agents both in vitro and in vivo. ${ }^{16}$ Cotreatment with $3 n$ protected both the white matter and the cortical plate against the insult (Figure 2). Furthermore, both doses ( 1 and $10 \mathrm{mg} / \mathrm{kg}$ ) of $\mathrm{A}, \mathrm{B}$ and $3 \mathbf{n}$ were neuroprotective, 50 while only the highest dose $(10 \mathrm{mg} / \mathrm{kg})$ of $\mathrm{C}$ yielded a significant protection in this model. Finally, in newborn mice, 3,3diphenylsubstituted 1,4-benzoxazine $3 \mathbf{n}$ proved to be more potent than benzoxazines A-C, making it a promising candidate as neuroprotective agent.
Table 3 Variation of the dienophile part: tandem oxidation-IEDDA reaction of aminophenol 1a with different enamines $2 \mathbf{a}-\mathbf{i}^{a}$

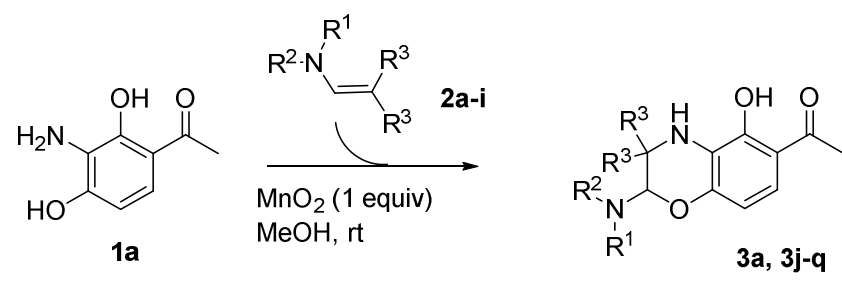

\begin{tabular}{|c|c|c|c|}
\hline Entry & Enamine $\mathbf{2}$ & Product 3 & $\begin{array}{l}\text { Yield } \\
(\%)^{b}\end{array}$ \\
\hline 1 & & & 75 \\
\hline
\end{tabular}

2<smiles>CC(C)=CN1CCOC1</smiles><smiles>CC(=O)c1ccc2c(c1O)NC(C)(C)C(N(C=O)C=CO)O2</smiles>

3<smiles>O=CCN(C=C1CCCCC1)CCO</smiles><smiles>C/C=C\CC12C=CCN(C=O)C1c1ccc(C(C)=O)c(O)c1N2</smiles>

4<smiles>O=CCN(C=C(c1ccccc1)c1ccccc1)CC=O</smiles>

2d<smiles>CC(=O)c1ccc2c(c1O)NC(c1ccccc1)(c1ccccc1)O2</smiles>

5<smiles>CN(/C=C(\c1ccccc1)C1OCCO1)CN(C)CC1OCCO1</smiles><smiles>CC(=O)c1ccc2c(c1O)NC(c1ccccc1)(c1ccccc1)OC2N(C)CC1OCCO1</smiles>

6<smiles>COCCNC=C(c1ccccc1)c1ccccc1</smiles><smiles>COCCNC1Oc2ccc(CC(=O)c3ccccc3)c(O)c2NC1c1ccccc1</smiles>

7<smiles>C(=C/c1ccccc1)\NCc1cccs1</smiles><smiles>CC(=O)c1ccc2c(c1O)NC(c1ccccc1)(c1ccccc1)O2</smiles>

8<smiles>COC(CNC=C(c1ccccc1)c1ccccc1)OC</smiles><smiles>COC(CNC1Oc2ccc(F)c(C(C)=O)c2NC1(c1ccccc1)c1ccccc1)OC</smiles>

9

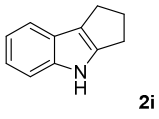

$60{ }^{a}$ Reactions were performed with 1 a $(0.5 \mathrm{mmol})$, enamines 2a-h $(0.75$ mmol) and $\mathrm{MnO}_{2}(0.5 \mathrm{mmol}, 1$ equiv) in $25 \mathrm{~mL}$ of deaerated $\mathrm{MeOH}$, at room temperature, for $3 \mathrm{~h} .1 .5$ equiv of morpholine was added to the bulk solution for generating the phenolate ion of 1a. ${ }^{b}$ Yields refer to chromatographically pure isolated products. ${ }^{c} 2.5 \mathrm{mmol}$ ( 5 equiv) of indole ${ }_{65} \mathbf{2} \mathbf{i}$ was necessary for the reaction. 
<smiles>CN(CC1OCCO1)C1Oc2ccc(C(=O)c3ccccc3)c(O)c2NC1(c1ccccc1)c1ccccc1</smiles><smiles>COCCNC1Oc2ccc(C(C)=O)c(O)c2NC1(c1ccccc1)c1ccccc1</smiles><smiles>O=C(c1ccccc1)c1ccc(NCc2ccccc2)c2c1NC1(CCCC1)CO2</smiles><smiles>CC(C)(C)C1COc2c(NCCc3ccccc3)ccc(C(=O)c3ccccc3)c2N1</smiles>

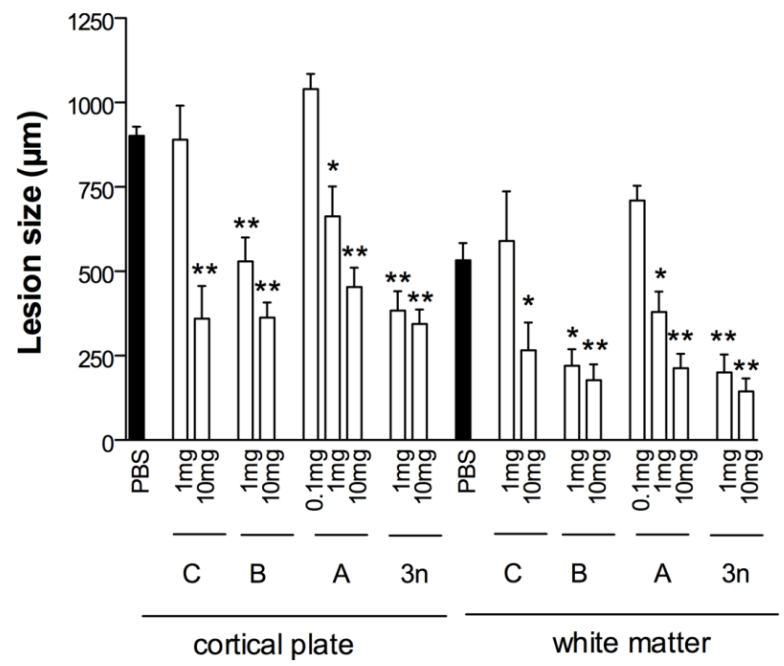

Fig. 2 In vivo biological evaluation in newborn mice. The histograms represent the mean length of the neocortical lesion in the sagittal fronto5 occipital axis \pm S.E.M. Asterisks indicate difference from control (* $\mathrm{P}<0.05, * * \mathrm{P}<0.01$ in ANOVA with Dunnet's multiple comparison test). PBS: control animals injected with $S$-bromo-willardiine into the cortex and white matter and i.p. with physiological saline; all the other experimental groups were cotreated with $S$-bromo-willardiine and the 10 selected drug at the doses indicated in $\mathrm{mg} / \mathrm{kg}$, i.p.

\section{Conclusions}

We have reported a successful use of in situ generated $o$-quinonemonoimine heterodienes for the regiospecific IEDDA reaction with enamine dienophiles. The possibility of introducing 15 variations in both cycloaddition partners led to highly substituted 1,4-benzoxazine cycloadducts with up to five elements of diversity. The reactions proceeded under mild conditions, at room temperature, with a good efficiency. The low toxicity, low cost, and ease of handling stoichiometric amount of activated $\mathrm{MnO}_{2}$, 20 combined with its commercial availability, are more attractive than the previously reported electrochemical conditions for the synthesis of derivatives at a preparative scale. By means of the current protocol, 3,3-diphenyl-substituted-1,4-benzoxazine 3n was identified as an effective neuroprotective agent in newborn 25 mice, suggesting that it could be a potential candidate for the treatment and prevention of cerebral palsy, together with probably other neurodegenerative disorders involving excitotoxicity as a pathomechanism.

\section{${ }_{30}$ Experimental section}

\section{General Information}

35 Analytical thin-layer chromatography was carried out on silica gel Macherey-Nagel Polygram SIL G/UV 254 (0.25 mm). Column chromatography was performed on Macherey-Nagel $\mathrm{Si}$ $60 \mathrm{M}$ silica gel $(40-63 \mu \mathrm{m})$.

Melting points were measured on a Köfler apparatus.

${ }_{45}{ }^{1} \mathrm{H}$ NMR and ${ }^{13} \mathrm{C}$ NMR spectra were performed on a Bruker AC-300 spectrometer operating at 300 and $75 \mathrm{MHz}$, respectively. Chemical shifts are expressed as $\delta$ units (part per million) downfield from TMS (tetramethylsilane). The measurements were carried out using the standard pulse sequences. The carbon

50 type (methyl, methylene, methine, or quaternary) was determined by DEPT experiments. ${ }^{1} \mathrm{H}$ and ${ }^{13} \mathrm{C}$ NMR spectra of all compounds are included in the supporting information as a proof of their identity.

High-resolution mass spectra (HRMS) were performed on a ${ }_{50}$ Bruker maXis mass spectrometer operating in positive ion mode by the "Fédération de Recherche" ICOA/CBM (FR2708) platform.

Chemicals were commercial products of the highest available ${ }_{60}$ purity and were used as supplied. 2-Aminophenol 1i and 2,3dihydro- $1 H$-cyclopent[b]indole $\mathbf{2 i}$ were commercially available compounds. Substituted $o$-aminophenol starting materials 1a-h were synthesized through previously reported procedures. ${ }^{7 \mathrm{c},} 17$ Enamine dienophiles 2a-h were prepared by standard methods ${ }_{65}$ from a mixture of aldehyde, appropriate amine (slight excess) and toluene- $p$-sulfonic acid (catalytic amount) heated at reflux, in benzene, for $18 \mathrm{~h}$, the water being continuously removed by means of a Dean-Stark separator.

General procedure for the tandem oxidation-IEDDA reaction of $o$-aminophenol derivatives $1 \mathrm{a}-\mathrm{i}$ with enamines $2 \mathrm{a}$-i

A solution of enamine $(0.75 \mathrm{mmol}, 1.5$ equiv $)$ in deaerated methanol $(5 \mathrm{~mL})$ was added to a solution of $o$-aminophenol derivative ( $0.5 \mathrm{mmol}, 1$ equiv) in deaerated methanol $(20 \mathrm{~mL})$ containing activated $\mathrm{MnO}_{2}(43.5 \mathrm{mg}, 0.5 \mathrm{mmol}, 1$ equiv) and 75 morpholine ( $66 \mu \mathrm{L}, 0.75 \mathrm{mmol}, 1.5$ equiv). The resulting mixture was stirred at room temperature for $3 \mathrm{~h}$, filtered off, and the filtrate was evaporated under reduced pressure at $30^{\circ} \mathrm{C}$. The residue was purified by column chromatography (toluene/acetone $95 / 5 \mathrm{v} / \mathrm{v}$ ) on silica gel to afford the desired 1,4-benzoxazine 80 derivative.

The above procedure is generally representative for all the 75 products shown in Tables 2 and 3. Any deviations from this protocol are specified in the footnotes of the tables.

[(R,S)-3,3-diethyl-5-hydroxy-2-morpholino-3,4-dihydro-2H1,4-benzoxazin-6-yl](methyl) methanone (3a)

${ }_{85}$ Pale yellow solid (125 mg, 75\%): $\mathrm{mp} 138-142^{\circ} \mathrm{C} ; R_{f}=0.35$ (toluene/acetone 98/2 v/v); ${ }^{1} \mathrm{H}$ NMR $\left(300 \mathrm{MHz}, \mathrm{CDCl}_{3}\right) \delta 0.82$ (t, $J=7.4 \mathrm{~Hz}, 3 \mathrm{H}), 0.97(\mathrm{t}, J=7.4 \mathrm{~Hz}, 3 \mathrm{H}), 1.40-2.00(\mathrm{~m}, 4 \mathrm{H})$, $2.59(\mathrm{~m}, 5 \mathrm{H}), 3.41(\mathrm{~m}, 2 \mathrm{H}), 3.70(\mathrm{~m}, 4 \mathrm{H}), 4.13(\mathrm{~s}, 1 \mathrm{H}), 4.57(\mathrm{~s}$, $1 \mathrm{H}), 6.45(\mathrm{~d}, J=8.8 \mathrm{~Hz}, 1 \mathrm{H}), 7.16(\mathrm{~d}, J=8.8 \mathrm{~Hz}, 1 \mathrm{H}), 12.83$ (s, $\left.{ }_{90} 1 \mathrm{H}\right) ;{ }^{13} \mathrm{C}\left\{{ }^{1} \mathrm{H}\right\}$ NMR $\left(75 \mathrm{MHz}, \mathrm{CDCl}_{3}\right) \delta 6.7,7.7,25.8,26.2,27.1$, 49.1, 55.4, 67.2, 95.7, 107.1, 113.3, 120.2, 120.8, 150.2, 151.3, 203.3; HRMS (ESI+) $\mathrm{m} / \mathrm{z}$ calcd for $\mathrm{C}_{18} \mathrm{H}_{26} \mathrm{~N}_{2} \mathrm{O}_{4} \quad[\mathrm{M}+\mathrm{H}]^{+}$ 335.1965 ; found 335.1966 . 
$[(R, S)-3,3-d i e t h y l-5-h y d r o x y-2-m o r p h o l i n o-3,4-d i h y d r o-2 H-$ 1,4-benzoxazin-6-yl] (cyclohexyl)methanone (3b)

Pale yellow solid (151 mg, 75\%): $\mathrm{mp} 152-156^{\circ} \mathrm{C} ; R_{f}=0.45$ (toluene/acetone 98/2 v/v); ${ }^{1} \mathrm{H} \mathrm{NMR}\left(300 \mathrm{MHz}, \mathrm{CDCl}_{3}\right) \delta 0.82$ $5(\mathrm{t}, J=7.4 \mathrm{~Hz}, 3 \mathrm{H}), 0.97(\mathrm{t}, J=7.4 \mathrm{~Hz}, 3 \mathrm{H}), 1.20-2.00(\mathrm{~m}, 14 \mathrm{H})$, $2.63(\mathrm{~m}, 2 \mathrm{H}), 3.26(\mathrm{t}, J=5.4 \mathrm{~Hz}, 1 \mathrm{H}), 3.42(\mathrm{~m}, 2 \mathrm{H}), 3.71(\mathrm{~m}$, $4 \mathrm{H}), 4.14(\mathrm{~s}, 1 \mathrm{H}), 4.57(\mathrm{~s}, 1 \mathrm{H}), 6.45(\mathrm{~d}, J=8.8 \mathrm{~Hz}, 1 \mathrm{H}), 7.22(\mathrm{~d}$, $J=8.8 \mathrm{~Hz}, 1 \mathrm{H}), 13.21(\mathrm{~s}, 1 \mathrm{H}) ;{ }^{13} \mathrm{C}\left\{{ }^{1} \mathrm{H}\right\} \mathrm{NMR}\left(75 \mathrm{MHz}, \mathrm{CDCl}_{3}\right) \delta$ 6.7, 7.7, 25.87, 25.89, 25.92, 27.1, 29.7, 29.8, 44.9, 49.1, 55.4, 10 67.1, 95.6, 106.9, 111.9, 112.3, 119.9, 120.4, 149.8, 152.0, 209.0; Anal. calcd for $\mathrm{C}_{23} \mathrm{H}_{34} \mathrm{~N}_{2} \mathrm{O}_{4}$ : C, 68.66; $\mathrm{H}, 8.46, \mathrm{~N}, 6.96$. Found: C, 68.60; H, 8.49, N, 6.94.

[(R,S)-3,3-diethyl-5-hydroxy-2-morpholino-3,4-dihydro-2H1,4-benzoxazin-6-yl] (isobutyl)methanone (3c)

15 Yellow solid (139 mg, 74\%): $\mathrm{mp} \quad 152-156^{\circ} \mathrm{C} ; \quad R_{f}=0.4$ (toluene/acetone 98/2 v/v); ${ }^{1} \mathrm{H} \mathrm{NMR}\left(300 \mathrm{MHz}, \mathrm{CDCl}_{3}\right) \delta 0.82$ (t, $J=7.4 \mathrm{~Hz}, 3 \mathrm{H}), 0.97$ (t, $J=7.4 \mathrm{~Hz}, 3 \mathrm{H}), 1.05$ (d, $J=7.2 \mathrm{~Hz}$, $6 \mathrm{H}) 1.49-2.00(\mathrm{~m}, 4 \mathrm{H}), 2.32(\mathrm{~m}, 1 \mathrm{H}), 2.61(\mathrm{~m}, 2 \mathrm{H}), 2.80(\mathrm{~d}, J=$ $6.9 \mathrm{~Hz}, 2 \mathrm{H}), 3.41(\mathrm{~m}, 2 \mathrm{H}), 3.70(\mathrm{~m}, 4 \mathrm{H}), 4.13(\mathrm{~s}, 1 \mathrm{H}), 4.56(\mathrm{~s}$, $201 \mathrm{H}), 6.43(\mathrm{~d}, J=8.9 \mathrm{~Hz}, 1 \mathrm{H}), 7.18(\mathrm{~d}, J=8.9 \mathrm{~Hz}, 1 \mathrm{H}), 13.08(\mathrm{~s}$, $1 \mathrm{H}) ;{ }^{13} \mathrm{C}\left\{{ }^{1} \mathrm{H}\right\}$ NMR $\left(75 \mathrm{MHz}, \mathrm{CDCl}_{3}\right) \delta 6.7,7.7,22.8,25.83$, 25.88, 27.1, 46.8, 49.1, 55.4, 67.2, 95.7, 106.9, 113.3, 120.26, 120.33, 150.0, 151.6, 205.5; Anal. calcd for $\mathrm{C}_{21} \mathrm{H}_{32} \mathrm{~N}_{2} \mathrm{O}_{4}$ : C, 67.02; H, 8.51, N, 7.45. Found: C, 66.90; H, 8.54, N, 7.40.

${ }_{25}[(\boldsymbol{R}, \boldsymbol{S})$-3,3-diethyl-2-morpholino-3,4-dihydro-2H-1,4benzoxazin-6-yl](methyl)methanone (3d)

White solid (108 mg, 68\%): $\mathrm{mp} 134-138^{\circ} \mathrm{C} ; R_{f}=0.25$ (toluene/acetone 95/5 v/v); ${ }^{1} \mathrm{H} \mathrm{NMR}\left(300 \mathrm{MHz}, \mathrm{CDCl}_{3}\right) \delta 0.81$ (t, $J=7.4 \mathrm{~Hz}, 3 \mathrm{H}), 0.93(\mathrm{t}, J=7.4 \mathrm{~Hz}, 3 \mathrm{H}), 1.51-1.61(\mathrm{~m}, 3 \mathrm{H})$, $301.70-1.90(\mathrm{~m}, 1 \mathrm{H}), 2.53(\mathrm{~s}, 3 \mathrm{H}), 2.58(\mathrm{~m}, 2 \mathrm{H}), 3.35(\mathrm{~m}, 2 \mathrm{H}), 3.65$ $(\mathrm{m}, 4 \mathrm{H}), 3.83(\mathrm{~s}, 1 \mathrm{H}), 4.52(\mathrm{~s}, 1 \mathrm{H}), 6.83(\mathrm{~d}, J=8.3 \mathrm{~Hz}, 1 \mathrm{H}), 7.22$ $(\mathrm{s}, 1 \mathrm{H}), 7.33(\mathrm{~d}, J=8.3 \mathrm{~Hz}, 1 \mathrm{H}) ;{ }^{13} \mathrm{C}\left\{{ }^{1} \mathrm{H}\right\}$ NMR $(75 \mathrm{MHz}$, $\left.\mathrm{CDCl}_{3}\right) \delta 6.7,7.5,26.18,26.22,27.0,49.2$, 55.7, 67.2, 95.1, 114.5, 114.7, 121.0, 130.4, 131.9, 149.3, 197.1; Anal. calcd for ${ }_{35} \mathrm{C}_{18} \mathrm{H}_{26} \mathrm{~N}_{2} \mathrm{O}_{3}$ : C, 67.92; H, 8.18, N, 8.80. Found: C, 67.81; H, 8.21, N, 8.77.

[(R,S)-3,3-diethyl-2-morpholino-3,4-dihydro-2H-1,4benzoxazin-6-yl]carbonitrile (3e)

White solid (103 mg, 68\%): $\mathrm{mp} 156-160^{\circ} \mathrm{C} ; R_{f}=0.35$ 40 (toluene/acetone 95/5 v/v); ${ }^{1} \mathrm{H}$ NMR $\left(300 \mathrm{MHz}, \mathrm{CDCl}_{3}\right) \delta 0.81$ (t, $J=7.4 \mathrm{~Hz}, 3 \mathrm{H}), 0.94$ (t, $J=7.4 \mathrm{~Hz}, 3 \mathrm{H}), 1.50-1.70(\mathrm{~m}, 3 \mathrm{H})$, 1.70-1.90 (m, 1H), $2.59(\mathrm{~m}, 2 \mathrm{H}), 3.31(\mathrm{~m}, 2 \mathrm{H}), 3.66(\mathrm{~m}, 4 \mathrm{H})$, $3.85(\mathrm{~s}, 1 \mathrm{H}), 4.53(\mathrm{~s}, 1 \mathrm{H}), 6.78(\mathrm{~s}, 1 \mathrm{H}), 6.83(\mathrm{~d}, J=8.3 \mathrm{~Hz}, 1 \mathrm{H})$, $6.99(\mathrm{~d}, J=8.3 \mathrm{~Hz}, 1 \mathrm{H}) ;{ }^{13} \mathrm{C}\left\{{ }^{1} \mathrm{H}\right\} \mathrm{NMR}\left(75 \mathrm{MHz} \mathrm{CDCl}_{3}\right) \delta 6.7$, 45 7.5, 26.4, 26.9, 49.1, 55.8, 67.1, 95.1, 103.5, 115.8, 117.7, 119.7, 123.6, 132.6, 148.5; Anal. calcd for $\mathrm{C}_{17} \mathrm{H}_{23} \mathrm{~N}_{3} \mathrm{O}_{2}$ : C, 67.77; $\mathrm{H}$, 7.64, N, 13.95. Found: C, 67.60; H, 7.66, N, 13.89.

[(R,S)-3,3-diethyl-2-morpholino-3,4-dihydro-2H-1,4benzoxazin-6-yl] (methyl) carboxylate (3f)

${ }_{50}$ White solid $(104 \mathrm{mg}, 62 \%): \mathrm{mp} 160-164^{\circ} \mathrm{C} ; R_{f}=0.4$ (toluene/acetone 95/5 v/v); ${ }^{1} \mathrm{H}$ NMR $\left(300 \mathrm{MHz}, \mathrm{CDCl}_{3}\right) \delta 0.81$ $(\mathrm{t}, J=7.4 \mathrm{~Hz}, 3 \mathrm{H}), 0.93(\mathrm{t}, J=7.4 \mathrm{~Hz}, 3 \mathrm{H}), 1.50-1.65(\mathrm{~m}, 3 \mathrm{H})$, 1.70-1.85 (m, 1H), $2.58(\mathrm{~m}, 2 \mathrm{H}), 3.35(\mathrm{~m}, 2 \mathrm{H}), 3.66(\mathrm{~m}, 4 \mathrm{H})$, $3.77(\mathrm{~s}, 1 \mathrm{H}), 3.87(\mathrm{~s}, 3 \mathrm{H}), 4.50(\mathrm{~s}, 1 \mathrm{H}), 6.81(\mathrm{~d}, J=8.3 \mathrm{~Hz}, 1 \mathrm{H})$, ${ }_{55} 7.26(\mathrm{~s}, 1 \mathrm{H}), 67.41(\mathrm{~d}, J=8.3 \mathrm{~Hz}, 1 \mathrm{H}) ;{ }^{13} \mathrm{C}\left\{{ }^{1} \mathrm{H}\right\} \mathrm{NMR}(75 \mathrm{MHz}$,
$\left.\mathrm{CDCl}_{3}\right) \delta 6.7,7.5,26.1,27.0,49.2,51.8,55.7,67.2,95.0,114.9$, $116.2,121.3,122.5,131.6,148.9,167.2$; Anal. calcd for $\mathrm{C}_{18} \mathrm{H}_{26} \mathrm{~N}_{2} \mathrm{O}_{4}$ : C, 64.67; H, 7.78, N, 8.38. Found: C, 64.70; H, 7.80, N, 8.38.

\section{$60[(R, S)-3,3-d i e t h y l-2-m o r p h o l i n o-3,4-d i h y d r o-2 H-1,4-$} benzoxazin-6-yl](phenyl)methanone (3g)

Yellow solid (114 mg, 60\%): $\mathrm{mp} 150-154^{\circ} \mathrm{C} ; \quad R_{f}=0.35$ (toluene/acetone 95/5 v/v); ${ }^{1} \mathrm{H}$ NMR $\left(300 \mathrm{MHz}, \mathrm{CDCl}_{3}\right) \delta 0.83$ $(\mathrm{t}, J=7.4 \mathrm{~Hz}, 3 \mathrm{H}), 0.94(\mathrm{t}, J=7.4 \mathrm{~Hz}, 3 \mathrm{H}), 1.50-1.7(\mathrm{~m}, 3 \mathrm{H})$, ${ }_{65} 1.82(\mathrm{~m}, 1 \mathrm{H}), 2.61(\mathrm{~m}, 2 \mathrm{H}), 3.38(\mathrm{~m}, 2 \mathrm{H}), 3.68(\mathrm{~m}, 4 \mathrm{H}), 3.83(\mathrm{~s}$, $1 \mathrm{H}), 4.54(\mathrm{~s}, 1 \mathrm{H}), 6.83(\mathrm{~d}, J=8.1 \mathrm{~Hz}, 1 \mathrm{H}), 7.15(\mathrm{~d}, J=8.2 \mathrm{~Hz}$, $1 \mathrm{H}), 7.17(\mathrm{~s}, 1 \mathrm{H}), 7.48(\mathrm{~m}, 2 \mathrm{H}), 7.57(\mathrm{~m}, 1 \mathrm{H}), 7.77(\mathrm{~d}, J=7.5 \mathrm{~Hz}$, $2 \mathrm{H}) ;{ }^{13} \mathrm{C}\left\{{ }^{1} \mathrm{H}\right\} \mathrm{NMR}\left(75 \mathrm{MHz}, \mathrm{CDCl}_{3}\right) \delta 6.7,7.6,26.3,27.0,49.2$, 55.7, 67.2, 95.1, 114.4, 116.5, 123.3, 128.0, 129.7, 130.2, 131.6, 70 132.0, 138.6, 149.1, 195.8; Anal. calcd for $\mathrm{C}_{23} \mathrm{H}_{28} \mathrm{~N}_{2} \mathrm{O}_{3}$ : C, 72.63; H, 7.37, N, 7.37. Found: C, 72.49; H, 7.40, N, 7.35.

\section{[(R,S)-3,3-diethyl-2-morpholino-3,4-dihydro-2H-1,4-} benzoxazin-6-yl](isobutyl) carboxamide (3h)

White solid (109 mg, 58\%): $\mathrm{mp} 198-202^{\circ} \mathrm{C} ; R_{f}=0.5$ (ethyl 75 acetate/petroleum ether $50 / 50 \mathrm{v} / \mathrm{v}) ;{ }^{1} \mathrm{H}$ NMR $\left(300 \mathrm{MHz}, \mathrm{CDCl}_{3}\right.$ ) $\delta 0.79(\mathrm{t}, J=7.4 \mathrm{~Hz}, 3 \mathrm{H}), 0.91(\mathrm{t}, J=7.4 \mathrm{~Hz}, 3 \mathrm{H}), 0.96(\mathrm{~d}, J=6.6$ $\mathrm{Hz}, 6 \mathrm{H}), 1.40-1.65(\mathrm{~m}, 3 \mathrm{H}), 1.70-1.90(\mathrm{~m}, 2 \mathrm{H}), 2.56(\mathrm{~m}, 2 \mathrm{H})$, $3.23(\mathrm{~m}, 1 \mathrm{H}), 3.34(\mathrm{~m}, 2 \mathrm{H}), 3.64(\mathrm{~m}, 4 \mathrm{H}), 3.95(\mathrm{~s}, 1 \mathrm{H}), 4.44(\mathrm{~s}$, $1 \mathrm{H}), 6.20(\mathrm{~s}, 1 \mathrm{H}), 6.77(\mathrm{~d}, J=8.2 \mathrm{~Hz}, 1 \mathrm{H}), 7.26(\mathrm{~s}, 1 \mathrm{H}), 6.98(\mathrm{~d}$, $\left.{ }_{80} J=8.2 \mathrm{~Hz}, 1 \mathrm{H}\right), 7.11(\mathrm{~s}, 1 \mathrm{H}) ;{ }^{13} \mathrm{C}\left\{{ }^{1} \mathrm{H}\right\} \mathrm{NMR}\left(75 \mathrm{MHz}, \mathrm{CDCl}_{3}\right) \delta$ 6.7, 7.6, 20.2, 26.2, 27.0, 28.6, 47.3, 49.2, 55.6, 67.2, 94.7, 114.56, 114.63, 116.8, 127.3, 132.1, 147.3, 167.6; Anal. calcd for $\mathrm{C}_{21} \mathrm{H}_{33} \mathrm{~N}_{3} \mathrm{O}_{3}$ : C, 67.20; H, 8.80, N, 11.20. Found: C, 66.98; H, $8.85, \mathrm{~N}, 11.12$.

\section{5 (R,S)-3,3-diethyl-2-morpholino-3,4-dihydro-2H-1,4- benzoxazine ( $3 i$ )}

White solid (84 mg, 62\%): $\mathrm{mp} 154-158^{\circ} \mathrm{C} ; \quad R_{f}=0.35$ (toluene/acetone 98/2 v/v); ${ }^{1} \mathrm{H}$ NMR $\left(300 \mathrm{MHz}, \mathrm{CDCl}_{3}\right) \delta 0.82$ $(\mathrm{t}, J=7.4 \mathrm{~Hz}, 3 \mathrm{H}), 0.93(\mathrm{t}, J=7.4 \mathrm{~Hz}, 3 \mathrm{H}), 1.40-1.9(\mathrm{~m}, 5 \mathrm{H})$, $902.61(\mathrm{~m}, 2 \mathrm{H}), 3.36(\mathrm{~m}, 2 \mathrm{H}), 3.66(\mathrm{~m}, 4 \mathrm{H}), 4.44(\mathrm{~s}, 1 \mathrm{H}), 6.53(\mathrm{~d}$, $J=7.6 \mathrm{~Hz}, 1 \mathrm{H}), 6.65-6.75(\mathrm{~m}, 2 \mathrm{H}), 6.81(\mathrm{~d}, J=7.6 \mathrm{~Hz}, 1 \mathrm{H})$; ${ }^{13} \mathrm{C}\left\{{ }^{1} \mathrm{H}\right\}$ NMR $\left(75 \mathrm{MHz}, \mathrm{CDCl}_{3}\right) \delta 6.8,7.6,26.2,27.1,49.3$, 55.6, 67.3, 94.1, 115.0, 115.2, 118.9, 120.6, 131.9, 144.6; Anal. calcd for $\mathrm{C}_{16} \mathrm{H}_{24} \mathrm{~N}_{2} \mathrm{O}_{2}: \mathrm{C}, 69.56 ; \mathrm{H}, 8.69, \mathrm{~N}, 10.14$. Found: C, ${ }_{95} 69.39 ; \mathrm{H}, 8.73, \mathrm{~N}, 10.11$.

[(R,S)-3,3-dimethyl-5-hydroxy-2-morpholino-3,4-dihydro-2H1,4-benzoxazin-6-yl] (methyl)methanone (3j)

Yellow solid (113 mg, 74\%): $\mathrm{mp} \quad 153-157^{\circ} \mathrm{C} ; R_{f}=0.35$ (toluene/acetone 95/5 v/v); ${ }^{1} \mathrm{H}$ NMR $\left(300 \mathrm{MHz}, \mathrm{CDCl}_{3}\right) \delta 1.26$ $100(\mathrm{~s}, 3 \mathrm{H}), 1.41(\mathrm{~s}, 3 \mathrm{H}), 2.59(\mathrm{~s}, 3 \mathrm{H}), 2.62(\mathrm{~m}, 2 \mathrm{H}), 3.36(\mathrm{~m}, 2 \mathrm{H})$, $3.69(\mathrm{~m}, 4 \mathrm{H}), 3.90(\mathrm{~s}, 1 \mathrm{H}), 4.52(\mathrm{~s}, 1 \mathrm{H}), 6.47(\mathrm{~d}, J=8.8 \mathrm{~Hz}, 1 \mathrm{H})$, $7.17(\mathrm{~d}, J=8.8 \mathrm{~Hz}, 1 \mathrm{H}), 12.84(\mathrm{~s}, 1 \mathrm{H}) ;{ }^{13} \mathrm{C}\left\{{ }^{1} \mathrm{H}\right\} \mathrm{NMR}(75 \mathrm{MHz}$, $\left.\mathrm{CDCl}_{3}\right) \delta 26.1,26.2,28.3,48.8,50.9,67.2,96.9,107.2,113.3$, 120.5, 120.9, 150.0, 151.5, 203.3; Anal. calcd for $\mathrm{C}_{16} \mathrm{H}_{22} \mathrm{~N}_{2} \mathrm{O}_{4}$ : C, 105 62.74; H, 7.19, N, 9.15. Found: C, 62.47; H, 7.21, N, 9.11.

[(R,S)-5-hydroxy-2-morpholino-3-spiro-1'-cyclohexyl-3,4dihydro-2H-1,4-benzoxazin-6-yl](methyl)methanone (3k)

Pale yellow solid (144 mg, 83\%): $\mathrm{mp} 68-72^{\circ} \mathrm{C} ; R_{f}=0.45$ (toluene/acetone 95/5 v/v); ${ }^{1} \mathrm{H}$ NMR $\left(300 \mathrm{MHz}, \mathrm{CDCl}_{3}\right) \delta 1.30$ $1101.90(\mathrm{~m}, 10 \mathrm{H}), 2.56(\mathrm{~s}, 3 \mathrm{H}), 2.58(\mathrm{~m}, 2 \mathrm{H}), 3.39(\mathrm{~m}, 2 \mathrm{H}), 3.66(\mathrm{~m}$, 
$4 \mathrm{H}), 4.27(\mathrm{~s}, 1 \mathrm{H}), 4.58(\mathrm{~s}, 1 \mathrm{H}), 6.42(\mathrm{~d}, J=8.8 \mathrm{~Hz}, 1 \mathrm{H}), 7.13(\mathrm{~d}$, $J=8.8 \mathrm{~Hz}, 1 \mathrm{H}), 12.81(\mathrm{~s}, 1 \mathrm{H}) ;{ }^{13} \mathrm{C}\left\{{ }^{1} \mathrm{H}\right\} \mathrm{NMR}\left(75 \mathrm{MHz}, \mathrm{CDCl}_{3}\right) \delta$ 21.3, 21.5, 25.4, 26.1, 34.2, 34.7, 48.9, 52.0, 67.1, 96.3, 107.0, $113.2,120.0,120.7,150.0,151.4,203.1$; Anal. calcd for ${ }_{5} \mathrm{C}_{19} \mathrm{H}_{26} \mathrm{~N}_{2} \mathrm{O}_{4}$ : C, 65.89; H, 7.51, N, 8.09. Found: C, 66.09; H, 7.51, N, 8.11.

[(R,S)-3,3-diphenyl-5-hydroxy-2-morpholino-3,4-dihydro-2H1,4-benzoxazin-6-yl](methyl) methanone (3l)

Pale yellow solid (143 mg, 60\%): mp $158-162^{\circ} \mathrm{C} ; R_{f}=0.45$ 10 (toluene/acetone 95/5 v/v); ${ }^{1} \mathrm{H}$ NMR (300 MHz, $\left.\mathrm{CDCl}_{3}\right) \delta 2.51$ (s, 3H), $2.54(\mathrm{~m}, 2 \mathrm{H}), 3.30-3.40(\mathrm{~m}, 6 \mathrm{H}), 4.98(\mathrm{~s}, 1 \mathrm{H}), 5.57(\mathrm{~s}$, $1 \mathrm{H}), 6.48(\mathrm{~d}, J=8.8 \mathrm{~Hz}, 1 \mathrm{H}), 7.10-7.44(\mathrm{~m}, 11 \mathrm{H}), 12.90$ (s, 1H); ${ }^{13} \mathrm{C}\left\{{ }^{1} \mathrm{H}\right\}$ NMR $\left(75 \mathrm{MHz}, \mathrm{CDCl}_{3}\right) \delta 26.1,49.1,63.3,67.0,94.7$, $107.2,113.4,120.0,121.8,126.7,126.8,126.98,127.02,128.0$, ${ }_{15} 128.4,144.0,145.8,150.0,151.9,203.2$; Anal. calcd for $\mathrm{C}_{30} \mathrm{H}_{26} \mathrm{~N}_{2} \mathrm{O}_{4}$ : C, 75.31; H, 5.44, N, 5.86. Found: C, 75.45; H, $5.45, \mathrm{~N}, 5.87$.

[(R,S)-3,3-diphenyl-5-hydroxy-2-( $N$-methyldioxolan)methyl amino-3,4-dihydro-2H-1,4-benzoxazin-6-yl](methyl)

20 methanone (3m)

Pale yellow solid (129 mg, 56\%): $\mathrm{mp} 144-148^{\circ} \mathrm{C} ; R_{f}=0.25$ (toluene/acetone 95/5 v/v); ${ }^{1} \mathrm{H} \mathrm{NMR}\left(300 \mathrm{MHz}, \mathrm{CDCl}_{3}\right) \delta 2.50$ $(\mathrm{s}, 3 \mathrm{H}), 2.58(\mathrm{~s}, 3 \mathrm{H}), 2.96(\mathrm{~m}, 2 \mathrm{H}), 3.71-3.80(\mathrm{~m}, 4 \mathrm{H}), 4.50(\mathrm{~s}$, $1 \mathrm{H}), 4.97(\mathrm{~s}, 1 \mathrm{H}), 5.93(\mathrm{~s}, 1 \mathrm{H}), 6.45(\mathrm{~d}, J=8.8 \mathrm{~Hz}, 1 \mathrm{H}), 7.10-7.50$ ${ }_{25}(\mathrm{~m}, 11 \mathrm{H}), 12.86(\mathrm{~s}, 1 \mathrm{H}) ;{ }^{13} \mathrm{C}\left\{{ }^{1} \mathrm{H}\right\} \mathrm{NMR}\left(75 \mathrm{MHz}, \mathrm{CDCl}_{3}\right) \delta 26.0$, 37.6, 57.8, 63.3, 64.5, 64.8, 94.2, 104.6, 107.2, 113.2, 120.0, $121.7,126.7,126.9,127.0,127.9,128.3,143.8,146.1,150.2$, 151.8, 203.1; Anal. calcd for $\mathrm{C}_{27} \mathrm{H}_{28} \mathrm{~N}_{2} \mathrm{O}_{5}: \mathrm{C}, 70.43$; $\mathrm{H}, 6.09, \mathrm{~N}$, 6.09. Found: C, 70.34; H, 6.11 N, 6.09.

$30[(\boldsymbol{R}, S)-3,3-d i p h e n y l-5-h y d r o x y-2-(2-m e t h o x y) e t h y l a m i n o-3,4-$ dihydro-2H-1,4-benzoxazin-6-yl](methyl)methanone (3n)

Pale yellow solid (136 mg, 65\%): $\mathrm{mp} 96-100^{\circ} \mathrm{C} ; R_{f}=0.35$ (ethyl acetate/petroleum ether 25/75 v/v); ${ }^{1} \mathrm{H}$ NMR (300 MHz, $\mathrm{CDCl}_{3}$ ) $\delta 2.60(\mathrm{~s}, 3 \mathrm{H}), 2.90-3.10(\mathrm{~m}, 2 \mathrm{H}), 3.24(\mathrm{~s}, 3 \mathrm{H}), 3.41(\mathrm{~s}, 2 \mathrm{H})$, $355.13(\mathrm{~s}, 1 \mathrm{H}), 5.80(\mathrm{~s}, 1 \mathrm{H}), 6.40(\mathrm{~d}, J=8.8 \mathrm{~Hz}, 1 \mathrm{H}), 7.10-7.60(\mathrm{~m}$, $11 \mathrm{H}), 12.97(\mathrm{~s}, 1 \mathrm{H}) ;{ }^{13} \mathrm{C}\left\{{ }^{1} \mathrm{H}\right\}$ NMR $\left(75 \mathrm{MHz}, \mathrm{CDCl}_{3}\right) \delta 26.3$, 44.9, 58.6, 62.4, 72.6, 89.5, 109.0, 113.5, 120.6, 121.4, 126.6, 127.0, 127.1, 128.2, 128.4, 143.0, 144.3, 146.9, 151.5, 203.5; HRMS (ESI+) $m / z$ calcd for $\mathrm{C}_{25} \mathrm{H}_{26} \mathrm{~N}_{2} \mathrm{O}_{4}[\mathrm{M}+\mathrm{H}]^{+} 419.1965$; 40 found 419.1963.

[(R,S)-3,3-diphenyl-5-hydroxy-2-(1-thienyl)methylamino-3,4dihydro-2H-1,4-benzoxazin-6-yl](methyl)methanone (3o)

White solid (157 mg, 69\%): $\mathrm{mp} 166-170^{\circ} \mathrm{C} ; R_{f}=0.3$ (toluene); ${ }^{1} \mathrm{H} \mathrm{NMR}\left(300 \mathrm{MHz}, \mathrm{CDCl}_{3}\right) \delta 2.56(\mathrm{~s}, 3 \mathrm{H}), 4.21(\mathrm{~m}, 2 \mathrm{H}), 5.22$ $45(\mathrm{~s}, 1 \mathrm{H}), 5.79(\mathrm{~s}, 1 \mathrm{H}), 6.44(\mathrm{~d}, J=8.8 \mathrm{~Hz}, 1 \mathrm{H}), 6.83(\mathrm{~d}, J=2.6 \mathrm{~Hz}$, 1H), $6.94(\mathrm{~m}, 1 \mathrm{H}), 7.10-7.50(\mathrm{~m}, 13 \mathrm{H}), 12.99(\mathrm{~s}, 1 \mathrm{H}) ;{ }^{13} \mathrm{C}\left\{{ }^{1} \mathrm{H}\right\}$ NMR $\left(75 \mathrm{MHz}, \mathrm{CDCl}_{3}\right) \delta 26.2,43.7,62.3,87.5,109.0,113.6$, $120.6,121.5,124.7,124.9,126.56,126.62,127.0,127.1,127.2$, 128.3, 128.4, 142.8, 143.6, 144.1, 146.7, 151.6, 203.4; Anal. 50 calcd for $\mathrm{C}_{27} \mathrm{H}_{24} \mathrm{~N}_{2} \mathrm{O}_{3} \mathrm{~S}$ : C, 71.05; H, 5.26, N, 6.14. Found: C, 70.83; H, 5.27, N, 6.12 .

[(R,S)-2-[(2,2-dimethoxy)ethylamino]-3,3-diphenyl-5hydroxy-3,4-dihydro-2H-1,4-benzoxazin-6-yl](methyl) methanone (3p)

${ }_{55}$ Pale yellow solid (117 mg, 52\%): $\mathrm{mp} 64-68^{\circ} \mathrm{C} ; R_{f}=0.3$ (toluene/acetone 95/5 v/v); ${ }^{1} \mathrm{H}$ NMR $\left(300 \mathrm{MHz}, \mathrm{CDCl}_{3}\right) \delta 2.40$ (broad s, 1H), $2.53(\mathrm{~s}, 3 \mathrm{H}), 2.85-3.10(\mathrm{~m}, 2 \mathrm{H}), 3.22(\mathrm{~s}, 3 \mathrm{H}), 3.24$ $(\mathrm{s}, 3 \mathrm{H}), 4.32(\mathrm{t}, J=5.7 \mathrm{~Hz}, 1 \mathrm{H}), 5.15(\mathrm{~s}, 1 \mathrm{H}), 5.77(\mathrm{~s}, 1 \mathrm{H}), 6.37$ $(\mathrm{d}, J=8.8 \mathrm{~Hz}, 1 \mathrm{H}), 7.11(\mathrm{~d}, J=8.8 \mathrm{~Hz}, 1 \mathrm{H}), 7.15-7.55(\mathrm{~m}, 10 \mathrm{H})$, ${ }_{60} 12.98(\mathrm{~s}, 1 \mathrm{H}) ;{ }^{13} \mathrm{C}\left\{{ }^{1} \mathrm{H}\right\} \mathrm{NMR}\left(75 \mathrm{MHz}, \mathrm{CDCl}_{3}\right) \delta 26.2,46.9$, 53.4, 54.2, 62.4, 89.3, 104.2, 109.0, 113.5, 120.6, 121.4, 126.6, 127.0, 127.1, 128.2, 128.5, 143.0, 144.2, 146.8, 151.6, 203.5; Anal. calcd for $\mathrm{C}_{26} \mathrm{H}_{28} \mathrm{~N}_{2} \mathrm{O}_{5}: \mathrm{C}, 69.64 ; \mathrm{H}, 6.25, \mathrm{~N}, 6.25$. Found: C, 69.49; H, 6.28, N, 6.24.

\section{5 [1-hydroxy-6H,11H-5a,10b-propanoindolo[2,3-b][1,4] benzoxazin-2-yl](methyl) methanone (3q)}

Pale pink solid (48 mg, 30\%): $\mathrm{mp} 144-148^{\circ} \mathrm{C} ; R_{f}=0.4$ (toluene/acetone 95/5 v/v); ${ }^{1} \mathrm{H}$ NMR $\left(300 \mathrm{MHz}, \mathrm{CDCl}_{3}\right) \delta 1.75$ $2.45(\mathrm{~m}, 6 \mathrm{H}), 2.51(\mathrm{~s}, 3 \mathrm{H}), 4.60($ broad s, $2 \mathrm{H}), 6.41(\mathrm{~d}, J=8.8 \mathrm{~Hz}$, $701 \mathrm{H}), 6.52(\mathrm{~d}, J=7.8 \mathrm{~Hz}, 1 \mathrm{H}), 6.76(\mathrm{t}, J=7.5 \mathrm{~Hz}, 1 \mathrm{H}), 7.05(\mathrm{t}, J=$ $7.5 \mathrm{~Hz}, 1 \mathrm{H}), 7.08(\mathrm{~d}, J=8.8 \mathrm{~Hz}, 1 \mathrm{H}), 7.16(\mathrm{~d}, J=7.5 \mathrm{~Hz}, 1 \mathrm{H})$, $12.7(\mathrm{~s}, 1 \mathrm{H}) ;{ }^{13} \mathrm{C}\left\{{ }^{1} \mathrm{H}\right\}$ NMR $\left(75 \mathrm{MHz}, \mathrm{CDCl}_{3}\right) \delta 22.7,26.2,38.3$, $41.5,70.5,107.2,113.6,120.6,121.5,124.7,124.9,126.56$, $126.62,127.0,127.1,127.2,128.3,128.4,142.8,143.6,144.1$, 75 146.7, 151.6, 203.4; Anal. calcd for $\mathrm{C}_{19} \mathrm{H}_{18} \mathrm{~N}_{2} \mathrm{O}_{3}: \mathrm{C}, 70.81 ; \mathrm{H}$, 5.59, N, 8.69. Found: C, 70.58; H, 5.63, N, 8.67.

\section{In vivo biological assay. Neonatal mice}

At postnatal day 5, Swiss mouse pups were anesthetized with isoflurane for intracerebral (ic) and intraperitoneal (ip) 80 injections. ${ }^{15,18}$ Intracerebral injections were performed using a 26gauge needle mounted on a calibrated microdispenser. The needle was inserted $2 \mathrm{~mm}$ under the external surface of scalp skin in the frontoparietal area of the right hemisphere $2 \mathrm{~mm}$ from the midline in the lateral-medial plane and $3 \mathrm{~mm}$ (in the rostro-caudal plane) 85 from the junction between sagittal and lambdoid sutures. Two 1 $\mu \mathrm{l}$ boluses were injected at a 30 -s interval.

Fifteen $\mu \mathrm{g} S$-bromo-willardiine (Tocris, Bristol, UK), diluted in phosphate buffer saline (PBS), were injected ic. Immediately following ic injection, 1 or $10 \mathrm{mg} / \mathrm{kg}$ compound $\mathrm{C}$ ( $\mathrm{n}=8$ and 6 , 90 respectively), 1 or $10 \mathrm{mg} / \mathrm{kg}$ compound $\mathrm{B}$ ( $\mathrm{n}=6$ and 15 , respectively), $0.1,1$ or $10 \mathrm{mg} / \mathrm{kg}$ compound $\mathrm{A}$ ( $\mathrm{n}=8,7$ and 9 , respectively), and 1 or $10 \mathrm{mg} / \mathrm{kg} 3 \mathrm{n}$ ( $\mathrm{n}=10$ in both groups) diluted in PBS containing 10\% dimethylsulfoxide (DMSO), were administered ip. Controls received ip PBS containing $10 \%$ 95 DMSO alone $(\mathrm{n}=19)$.

Five days later, the surviving pups were sacrificed and brains fixed in formalin. Coronal serial sections, $15 \mu \mathrm{m}$ thick, were cut and every third section was stained with cresyl-violet. Brain was completely and serially sectioned from the frontal pole to the 100 occipital lobes. In theory, neocortical and white matter lesions can be defined by the maximal length of three orthogonal axes: the lateral-medial axis (in a coronal plane), the radial axis (also in a coronal plane, from the pial surface to the lateral ventricle) and the fronto-occipital axis (in a sagittal plane). Due to the difficulty of 105 accurately evaluating the degree of damage to neurons in neocortical layers in the epicenter of the lesion focus, the radial axis did not appear as an objective measure of the lesion size. In previous studies, ${ }^{15,18}$ we had shown an excellent correlation between the maximal size of the radial and fronto-occipital diameters of the 110 excitotoxic lesions. Based on these observations, we serially sectioned the entire brain in the coronal plane. This permitted an accurate and reproducible determination of the maximal sagittal 
fronto-occipital diameter (which is equal to the number of sections where the lesion was present multiplied by $15 \mu \mathrm{m}$ ) and was as an index of the volume of the lesion. Statistical analyses were performed by analysis of variance (ANOVA) with Dunnet's 5 multiple comparison of means test. Results were expressed as means \pm S.E.M.

\section{Acknowledgements}

We thank CNRS, Inserm together with Paris Descartes and Paris Diderot Universities for financial support.

\section{${ }_{10}$ Notes and references}

${ }^{a}$ UMR 8638 CNRS-Université Paris Descartes, Sorbonne Paris Cité, Faculté de Pharmacie de Paris, 4 avenue de l'Observatoire, 75270 Paris cedex 06, France. Fax: +33144073588; Tel: +33153739646; E-mail: martine.largeron@parisdescartes.fr

${ }_{15}^{b}$ Inserm, U 1141, F-75019 Paris, France

${ }^{c}$ Université Paris Diderot, Sorbonne Paris Cité,UMRS 1141, F-75019 Paris, France

$\dagger$ Electronic Supplementary Information (ESI) available: ${ }^{1} \mathrm{H}$ and ${ }^{13} \mathrm{C} N M R$ spectra for benzoxazine derivatives 3a-q. See DOI: 10.1039/b000000x/

201 For recent reviews on biologically active 1,4-benzoxazine derivatives, see: (a) B. Achari, S. B. Mandal, P. K. Dutta, C. Chowdhury, Synlett, 2004, 14, 2449 and references therein; (b) F. A. Macias, D. Marin, A. Oliveros-Bastidas, J. M. G. Molinillo, Nat. Prod. Rep. 2009, 26, 478.

252 For selected recent examples, see: (a) P. Stefanic Anderluh, M. Anderluh, J. Ilas, J. Mravljak, M. Sollner Dolenc, M. Stegnar, D. Kikelj, J. Med. Chem. 2005, 48, 3110; (b) F. Schiaffella, A. Macchiarulo, L. Milanese, A. Vecchiarelli, G. Costantino, D. Pietrella, R. Fringuelli, J. Med. Chem. 2005, 48, 7658; (c) J. Ilas, Z.

30 Jakopin, T. Borstnar, M. Stegnar, D. Kikelj, J. Med. Chem. 2008, 51, 5617; (d) E. N. Koini, P. Papazafiri, A. Vassilopoulos, M. Koufaki, Z. Horvath, I. Koncz, L. Virag, G. J. Papp, A. Varro, T. Calogeropoulou, J. Med. Chem. 2009, 52, 2328; (e) X. Li, N. Liu, H. Zhang, S. E. Knudson, R. A.Slayden, P. J. Tonge, Bioorg. Med. Chem. Lett. 2010, 20, 6306; (f) T. Bouyssou, C. Hoenke, K. Rudolf, P. Lustenberger, S. Pestel, P. Sieger, R. Lotz, C. Heine, F. H. Büttner, A. Schnapp, I. Konetzki, Bioorg. Med. Chem. Lett. 2010, 20, 1410; (g) A. Wang, C.P. Prouty, P.D. Pelton, M. Yong, K. T. Demarest, W. V. Murray, G. -H. Kuo, Bioorg. Med. Chem. Lett. 2010, 20, 1432; (h) S. M. Bromidge, R. Arban, B. Bertani, S. Bison, M. Borriello, P. Cavanni, G. Dal Forno, R. Di-Fabio, D. Donati, S. Fontana, M. Gianotti, L. J. Gordon, E. Granci, C. P. Leslie, L. Moccia, A. Pasquarello, I. Sartori, A. Sava, J. M. Watson, A.Worby, L. Zonzini, V. Zucchelli, J. Med. Chem. 2010, 53, 5827; (i) M. Ilic, C. Kontogiorgis, D. Hadjipavlou-Litina, J. Ilas, D. Kikelj, Bioorg. Med. Chem. Lett. 2011, 21, 4705; (j) A. N. Matralis, M. G. Katselou, A. Nikitakis, A. P. Kourounakis, J. Med. Chem. 2011, 54, 5583; (k) V. P. Patil, V. L. Markad, K. M. Kodam, S. B. Waghmode, Bioorg. Med. Chem. Lett. 2013, 23, 6259; (l) G. Sonia, K. K. Thachil, M. K.

$50 \quad$ Parameswaran, R. T. Kochupappy, Med. Chem. Res. 2014, 23, 1320.

3 For a selected report on the synthesis of 1,4-benzoxazine derivatives, see: J. Ilas, P. Stefanic Anderluh, M. Sollner Dolenc, D. Kikelj, Tetrahedron, 2005, 61, 7325 and references therein.

4 For selected recent examples, see: (a) B. Gabriele, G. Salerno, L.

55 Veltri, R. Mancuso, Z. Li, A. Crispini, A. Bellusci, J. Org. Chem. 2006, 71, 7895; (b) X. Xu, L. Liang, J. Liu, J. Yang, L. Mai, Y. Li, Tetrahedron Lett. 2009, 50, 57; (c) Z. Liu, Y. Chen, Tetrahedron Lett. 2009, 50, 3790; (d) R. K. Rao, A. B. Naidu, G. Sekar, Org. Lett. 2009, 11, 192; (e) D. Chen, G. Shen, W. Bao, Org. Biomol. Chem. 2009, 7, 4067; (f) S. Bhadra, L. Adak, S. Samanta, A. K. M. Maidul Islam, M. Mukherjee, B. C. Ranu, J. Org. Chem. 2010, 75, 8533; (g) F. Melkonyan, A. Topolyan, A. Karchava, M. Yurovskaya, Tetrahedron, 2011, 67, 6826; (h) K. Brahma, A. Kumar Sasmal, C. Chowdhury, Org. Biomol. Chem. 2011, 9, 8422; (i) K. E. O. Ylijoki, 65 E. P. Kündig, Chem. Commun. 2011, 47, 10608; (j) R. K. Rao, G.
Sekar, Tetrahedron Asym. 2011, 22, 948; (k) J. S. Cannon, A. Olson, L. E. Overman, N. S. Solomon, J. Org. Chem. 2012, 77, 1961; (l) J. W. Zhang, Q. Cai, Q. Gu, X. -X. Shi, S. -L. You, Chem. Commun. 2013, 49, 7750; $(m)$ M. Nagarjuna Reddy, K. C. Kumara Swamy, Org. Biomol. Chem. 2013, 11, 7350; (n) B. Liu, M. Yin, H. Gao, W. Wu, H. Jiang, J. Org. Chem., 2013, 78, 3009.

5 For selected examples, see: (a) K. C. Nicolaou, Y. L. Zhong, P. S. Baran, P. S. Angew. Chem. Int. Ed. 2000, 39, 622; (b) K. C. Nicolaou, K. Sugita, P. S. Baran, Y. L. Zhong, Angew. Chem. Int. Ed. 2001, 40, 207; (c) K. C. Nicolaou, P. S. Baran, Y. L. Zhong, K. Sugita, J. Am. Chem. Soc. 2002, 124, 2212; (d) K. C. Nicolaou, K. Sugita, P. S. Baran, Y. L. Zhong, J. Am. Chem. Soc. 2002, 124, 2221; (e) M. Largeron, A. Neudörffer, M. Vuilhorgne, E. Blattes, M. -B. Fleury, Angew.Chem. Int. Ed. 2002, 41, 824; (f) J. Wolfer, T. Bekele,

80 C. J. Abraham, C. Dogo-Isonagie, T. Lectka, Angew. Chem. Int. Ed. 2006, 45, 7398; (g) J. -F. Bower, P. Szeto, T. Gallagher, Org. Lett. 2007, 9, 3283; (h) J. Iqbal, N. D. Tangellamudi, B. Dulla, S. Balasubramanian, Org. Lett. 2012, 14, 552; (i) M. S. Deshmukh, B. Das, N. Jain, RSC Adv. 2013, 3, 22389; (j) S. K. Singh, A. K. Bajpai, R. Saini, Tetrahedron Lett. 2013, 54, 7132; (k) L. Chouguiat, R. Boulcina, B. Carboni, A. Demonceau, A. Debache, Tetrahedron Lett. 2014, 55, 5124; (l) G. Choudhary, R. T. Naganaboina, R. K. Peddinti, RSC Adv. 2014, 4, 17969.

6 (a) W. M. Dai, X. Wang, C. Ma, Tetrahedron 2005, 61, 6879; (b) G. $90 \quad$ Feng, J. Wu, W. M. Dai, Tetrahedron 2006, 62, 4635; (c) Xing, X.; Wu, J.; Feng, G.; Dai, W. M. Tetrahedron 2006, 62, 6774; (d) E. Zhang, T. Xu, D. Wang, T. Huang, M. Yuan, J. Li, Y. Zou, RSC Adv. 2014, 4, 10022.

7 (a) E. Blattes, M. -B. Fleury, M. Largeron, J. Org. Chem. 2004, 69, 882; (b) D. Xu, A. Chiaroni, M. Largeron, Org. Lett. 2005, 7, 5273; (c) D. Xu, A. Chiaroni, M. -B. Fleury, M. Largeron, J. Org. Chem. 2006, 71, 6374.

8 E. Blattes, B. Lockhart, P. Lestage, L. Schwendimann, P. Gressens, M. -B. Fleury, M. Largeron, J. Med. Chem. 2005, 48, 1282.

1009 (a) A. Mc Killop, T. S. B. Sayer, J. Org. Chem. 1976, 41, 1079; (b) D. N. Nicolaides, R. Wajih Awad, E. A. Varella, J. Heterocyclic Chem. 1996, 33, 633; (c) D. N. Nicolaides, C. BezergiannidouBalouctsi, R. Wajih Awad, K. E. Litinas, E. Malamidou-Xenikaki, A. Terzis, C. P. Raptopoulou, J. Org. Chem. 1997, 62, 499.

10510 (a) H. W. Heine, B. J. Barchiesi, E. A. Williams, J. Org. Chem. 1984, 49, 2560; (b) D. S. C. Black, D. C.; Craig, H. W. Heine, N. Kumar, E. A. Williams, Tetrahedron Lett. 1987, 28, 6691; (c) H. W. Heine, C. Olsson, J. D. Bergin, J. B. Foresman, E. A. Williams, J. Org. Chem. 1987, 52, 97; (d) H. W. Heine, W. C. Schairer, J. A. Suriano, E. A. Williams, Tetrahedron, 1988, 44, 3181; (e) H. W. Heine, J. A Suriano, C. Winkel, A. Burik, C. M. Taylor, E. A. Williams, J. Org. Chem. 1989, 54, 5926; (f) G. Desimoni, G. Faita, P. P. Righeti, Tetrahedron, 1991, 47, 5857; $(g)$ H. W. Heine, D. K. Williams, J. L. Rutherford, J. Ramphal, E. A. Williams, Heterocycles, 1993, 35, 1125; (h) H. W. Heine, M. G. La Porte, R. H. Overbaugh, E. A. Williams, Heterocycles, 1995, 40, 743.

11 N. Bodipati, R. K. Peddinti, Org. Biomol. Chem. 2012, 10, 1958.

12 For reviews, see: (a) R. J. K. Taylor, M. Reid, J. Foot, S. A. Raw, Acc. Chem. Res. 2005, 38, 851 and references therein; (b) V. Jeena, R. S. Robinson, RSC Adv. 2014, 4, 40720 and references therein.

13 E. Blattes, M. -B. Fleury, M. Largeron, Electrochim. Acta 2005, 50, 4902.

14 For selected examples, see: (a) Y. Omote, A. Tomotake, C. Kashima, C. J. Chem. Soc. Perkin Trans 1 1988, 1988, 151; (b) M. S. South, L. Terri, M. D. Jakuboski, M. D. Westmeyer, D. R. Dukersherer, J. Org. Chem. 1996, 61, 8921; (c) R. C. Boruah, S. Ahmed, U. Sharma, J. S. Sandhu, J. Org. Chem. 2000, 65, 922; (d) R. M. Jones, C. Selenski, T. R. R. Pettus, J. Org. Chem., 2002, 67, 6911; (e) V. N. Kozhevnikov, D. N. Kozhevnikov, T. V. Nikitina, V. L. Rusinov, O. N. Chupakhin, M. Zabel, B. König, J. Org. Chem. 2003, 68, 2882; (f) D. R. Soenen, J. M. Zimpleman, D. L. Boger, J. Org. Chem. 2003, 68, 3593.

15 (a) S. L. Tahraoui, S. Marret, C. Bodénant, P. Leroux, M. A. Dommergues, P. Evrard, P. Gressens, Brain Pathol. 2001, 11, 56 ; (b) 135 I. Husson, B. Mesplès, P. Bac, J. Vamecq, P. Evrard, P. Gressens, 
Ann. Neurol. 2002, 51, 82 ; (c) I. Sfaello, O. Baud, A. Arzimanoglou, P. Gressens, Neurobiol. Dis. 2005, $20,837$.

16 (a) M. Largeron, B. Lockhart, B. Pfeiffer, M. -B. Fleury, J. Med. Chem. 1999, 42, 5043; (b) M. Largeron, B. Mesples, P. Gressens, R.

5 Cechelli, M. Spedding, A. Le Ridant, M. -B. Fleury, Eur. J. Pharmacol. 2001, 424, 189.

17 (a) H. Aichaoui, I. Lesieur, J. -P. Hénichart, Synthesis 1990, 8, 679 ; (b) R. Lok, R. E. Leone, A. J. Williams, J. Org. Chem. 1996, 61, 3289.

1018 (a) S. Marret, R. Mukendi, J. F. Gadisseux, P. Gressens, P. Evrard, J. Neuropathol. Exp. Neurol. 1995, 54, 358; (b) P. Gressens, S. Marret, J. M. Hill, D. E. Brenneman, I. Gozes, I. Fridkin, P. Evrard, J. Clin. Invest., 1997, 100, 390.

15 